\title{
RobotFusion: Grasping with a Robotic Manipulator via Multi-view Reconstruction
}

\author{
Daniele De Gregorio ${ }^{(凶)}$, Federico Tombari, and Luigi Di Stefano \\ DISI, University of Bologna, Bologna, Italy \\ \{daniele.degregorio3, federico.tombari, luigi.distefano\}@unibo.it
}

\begin{abstract}
We propose a complete system for 3D object reconstruction and grasping based on an articulated robotic manipulator. We deploy an RGB-D sensor as an end effector placed directly on the robotic arm, and process the acquired data to perform multi-view $3 \mathrm{D}$ reconstruction and object grasping. We leverage the high repeatability of the robotic arm to estimate 3D camera poses with millimeter accuracy and control each of the six sensor's DOF in a dexterous workspace. Thereby, we can estimate camera poses directly by robot kinematics and deploy a Truncated Signed Distance Function (TSDF) to accurately fuse multiple views into a unified $3 \mathrm{D}$ reconstruction of the scene. Then, we propose an efficient approach to segment the sought objects out of a planar workbench as well as a novel algorithm to automatically estimate grasping points.
\end{abstract}

Keywords: Grasp $\cdot$ Manipulation $\cdot$ Reconstruction

\section{Introduction}

Object recognition and 3D pose estimation are key tasks in industrial applications requiring autonomous robots to understand the surroundings and pursue grasping and manipulation [14]. Indeed, manipulation mandates estimation of the 6 Degree-Of-Freedom (6DOF) pose (position and orientation) of the objects with respect to the base coordinate system of the robot. This pose estimation should be not only robust to clutter, occlusion and sensor noise, but also efficient to avoid slowing down the manipulation process.

Most object recognition and pose estimation algorithms rely on matching $2 \mathrm{D}$ or $3 \mathrm{D}$ features between off-line 3D models (either sets of $3 \mathrm{D}$ scans or CAD models) and scene measurements in the form of depth or RGB-D images. In particular, exploitation of color and depth cues from RGB-D images through suitable integration of texture-based and 3D features can yield quite remarkable performance across a variety of benchmark RGB-D datasets [1,24].

Although providing compelling results on standard benchmarks, the above mentioned multi-stage, multi-modal, feature-based pipelines turn out unsuited

Electronic supplementary material The online version of this chapter (doi:10. 1007/978-3-319-49409-8_54) contains supplementary material, which is available to authorized users. 
to practical real-time industrial applications due to exceedingly slow execution times. Furthermore, due to reliance on a single vantage point, these approaches may fail when the sought objects are captured under high levels of occlusion [2] and/or their shape and texture do not appear distinctive enough in the chosen view. Finally, an additional nuisance that may hinder the performance of such approaches is represented by the high sensor noise affecting the depth frame provided as as input to the algorithms, which tends to distort significantly the 3D surfaces and often cause holes and artifacts [13].

In this paper, we investigate on the use of an RGB-D sensor mounted on top of a robotic arm to explore the surrounding environment in order to gather and process together RGB-D frames taken from different vantage points. This approach holds the potential to tackle the aforementioned issues inherent to perception from a single viewpoint, as also highlighted by the recent multi-view object recognition approach of Faulhammer et al. [7], who show how multi-view information can increase recognition accuracy and robustness by the integration of features from different viewpoints. In our work, we aim at 3D object reconstruction and grasping within a typical industrial robotics environment, namely a robotic arm performing pick-and-place operations on objects laying on planar surfaces such as workbenches, conveyor belts or positioners. The only working assumptions underpinning our approach consist in the robot being endowed with a highly accurate and repeatable encoder system (as it is typically the case of industrial robotic arms) and in objects laying on a plane. In these settings, we leverage on the high accuracy of robot encoders to automatically fuse together different views. First, at each vantage point we collect and fuse together multiple frames within a voxelized 3D representation, so to smooth out 3D data without distorting the underlying object geometries. Then, the views taken from different vantage points are merged together into a Truncated Signed Distance Function (TSDF) representation: we dub our approach Robot Fusion on account of its affinity to the well-known Kinect Fusion system [12]. Successively, we introduce a segmentation approach based on the planar assumption that allows for quick computation of grasping points without the need to carry out object recognition. Finally, the obtained grasping points are deployed to perform grasping via the robotic gripper mounted on the arm.

The novel contributions of our paper thus concern: (i) the Robot Fusion approach to reconstruct 3D objects by leveraging on robot encoders and TSDF representations, (ii) an algorithm to segment objects out of a planar surface, (iii) an algorithm to compute grasping points from surfaces without requiring any previous object recognition step. We report experimental results that demonstrate the effectiveness and accuracy of the proposed object reconstruction and segmentation stages, as well as the ability to attain good grasping points that enable to perform grasping successfully in cluttered scenes comprising several objects of diverse shapes.

\section{Related Work}

On-line fusion of the range images gathered by a moving sensor to achieve 3D reconstruction of the environment is a key task for RGB-D SLAM (Simultaneous 
Localization and Mapping) frameworks. Among prominent approaches, Kinect Fusion [12,23] attains a highly accurate 3D reconstruction of the environment by fusing the depth measurements taken from different viewpoints alongside with camera movement into an occupancy map, i.e. a discrete voxel grid where each cell may either be void or contain the distance from the nearest surface via a Truncated Signed Distance Function (TSDF).

Besides mapping, the other key task in SLAM frameworks consists in sensor localization. Purposely, the Iterative Closest Point (ICP) algorithm is widely deployed to align the current depth image with respect to either the previous one or the global map. However, due to the algorithm allowing to estimate successfully small motions only, ICP is prone to failure when the incoming frames get processed at a pace that turns out too slow compared to sensor movements. To overcome this issue, sparse matching of $2 \mathrm{D} / 3 \mathrm{D}$ features or dense, direct matching of image intensities may be deployed to coarsely align the current depth image prior to running ICP [5]. Several other methods, such as e.g. [10], rely on optimization of a global cost function to estimate all sensor poses coherently. Although very effective, these approaches are unsuited to real-time industrial robotics due to excessive running times, such as e.g. several minutes [10].

Differently, we address the SLAM localization task by relying on the high accuracy and repeatability of the robot encoders as well as on forward kinematics, as explained in details in Sect. 3.2. This leads to a fast and highly accurate 3D reconstruction step which is conducive to estimate a reliable set of grasping points on the reconstructed surfaces in absence of any information concerning the types of objects present in the scene. Indeed, estimating a set of contact point providing a stable grasp [11] based only on 3D geometry mandates very accurate $3 \mathrm{D}$ reconstruction of surfaces.

As for approaches aimed at grasping and manipulation based on grasp point detection, methods that try to estimate grasp points on unknown objects from a single range image have been proposed in $[16,25]$. These works are based on edge analysis and estimate the approach position of the gripper with respect to the target object (i.e., no estimation of the individual grasp points). Unfortunately, these methods can deal with thin objects or thin object parts only in scenes with low occlusion levels, both constraint due to the inherent limitations of relying on a single viewpoint already described in Sect.1. Another issue with these approaches is that the grasp configuration is computed for grippers with a simple geometry, like parallel grippers. Indeed, as in [16], using a complex gripper, like a five fingered anthropomorphic hand, requires shaping it as a parallel gripper to achieve successful grasp.

Another possible approach consists in estimating directly the grasp points on the object surface and compute, via inverse kinematics, the position and orientation of the gripper. Saut et al. [20] describes how it can be hard to achieve a grasp configuration for a multi-finger anthropomorphic hand in realtime, and similar conclusions are drawn in [8]. Thus, these methods are suitable to autonomous grasping based on offline estimation of grasp points on known objects, an object recognition stage to detect object instances and associated poses, a final strategy to choose automatically the optimal grasp configuration between a pre-defined list. 


\section{Proposed Method}

This Section describes in detail the main stages of the proposed system. As already mentioned, our approach relies on typical assumptions for industrial manipulation and pick-and-place, i.e. the presence of a high precision industrial robotic arm and a planar workbench holding objects.

In Subsect.3.1 we illustrate how forward and inverse kinematics can be employed to accurately compute the camera 6 DOF pose with respect to the robot main coordinate system regardless of the mounting point used on the robotic arm, this effectively replacing the visual data-based localization necessary to perform SLAM reconstruction of the scene. Then, we show how to attain accurate multi-view 3D reconstruction by fusing together range images within a voxelized TSDF representation. In Subsect. 3.2, we propose a segmentation approach based on a novel plane extraction method, dubbed HeightMap, which estimates all scene planes orthogonal to a given gravity vector. This results in a small and predictable computational time, which compares favorably to RANSAC-based plane fitting and allows for segmenting effectively the individual objects from the previously obtained 3D reconstruction. Finally, in Subsect. 3.3, we describe our proposed method for grasp points extraction from the 3D surface of segmented objects, which does not require an explicit object recognition stage. In particular, grasp points and grasp approach position are computed by leveraging on the object Canonical Reference Frame and so to avoid collisions between the robotic arm and the environment.

\subsection{Multi-view Reconstruction via RobotFusion}

We assume to deploy an industrial robot with high accuracy and repeatability (i.e. $\approx 0.05 \mathrm{~mm}[9]$ ). High accuracy means that we can impose a 6 -DOF end effector pose ${ }^{0} \mathbf{T}_{\mathbf{E E}}$ with high precision in a dexterous workspace:

$$
{ }^{0} T_{E E}=\left[\begin{array}{cc}
{ }^{0} R_{E E} & { }^{0} \mathbf{p}_{E E} \\
0 & 1
\end{array}\right]
$$

In our system, the end effector is an RGB-D camera (i.e. an Asus Xtion) with a 3D-printed housing, this yielding an unknown transformation between the robot wrist and the $3 \mathrm{D}$ coordinate system attached to the camera (i.e. the camera coordinate system):

$$
{ }^{0} T_{E E}={ }^{0} T_{W} \cdot{ }^{W} T_{E E}
$$

While ${ }^{0} \mathbf{T}_{\mathbf{W}}$ is the result of a simple application of forward kinematic equations on the target robot, ${ }^{\mathbf{W}} \mathbf{T}_{\mathbf{E E}}$ is estimated during an offline calibration stage by means of a fiducial marker having a known $3 \mathrm{D}$ pose, ${ }^{0} \mathbf{T}_{\mathbf{M}}$, in the main robot coordinate system. To accomplish this, we use an Augmented Reality Framework [18] to estimate the pose of the fiducial marker w.r.t. the camera coordinate system: ${ }^{\mathbf{C a m}} \mathbf{T}_{\mathbf{M}}={ }^{\mathbf{E}} \mathbf{T}_{\mathbf{M}}$, thereby closing the loop in the equation

$$
{ }^{0} T_{W} \cdot{ }^{W} T_{E E} \cdot{ }^{E E} T_{M}={ }^{0} T_{M}
$$




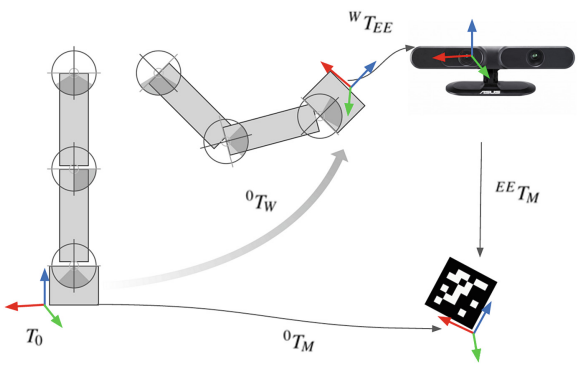

Fig. 1. Kinematics chain to compute the transformation between the wrist and the camera $\left({ }^{W} T_{E E}\right)$.

in order to estimate the unknown transformation between the wrist and the camera

$$
{ }^{W} T_{E E}={ }^{W} T_{0} \cdot{ }^{0} T_{M} \cdot{ }^{M} T_{E E}
$$

Now, each point, $p_{i}$, belonging to the point cloud $\mathcal{P}=\left\{p_{1}, \ldots, p_{n}\right\}$ acquired by the RGB-D sensor can be expressed as:

$$
{ }^{0} p_{i}={ }^{0} T_{E E} \cdot{ }^{E E} p_{i}
$$

Hence, for each new view gathered alongside robot movement, we can directly obtain a point cloud registered into the main robot coordinate system without the need to estimate any alignment transformation with respect to the previously acquired clouds (Fig. 1).

A 3D object reconstruction algorithm needs multiple range images from different viewpoints to build an unified representation of the surface of the target object. State-of-the-art SLAM techniques pursuing accurate and dense scene reconstruction, such as e.g. [4,12], first localize the camera and then merge each newly acquired range image into a global representation of the environment based on a Truncated Signed Distance Function (TSDF) defined on a voxel grid. Therein, each voxel either is void or stores the signed distance from its center to the nearest surface.

We adopt the same TSDF representation. However, as described, we do not need to carry out an explicit camera localization step as we can seamlessly integrate depth measurements into a global voxel grid by deploying robot kinematics. It is worthwhile pointing out that the use of a TSDF-based representation allows our system to fuse together seamlessly not only acquisitions from diverse viewpoints, but multiple range images taken from the same vantage point alike. The latter process turns out highly effective to counteract the significant amount of noise that typically affects the RGB-D data acquired by consumer depth cameras.

To minimize the amount of calculations, we update only the voxels visible from the current vantage point (view frustum) rather than the entire grid $^{1}$.

\footnotetext{
${ }^{1}$ https://github.com/sdmiller/cpu_tsdf.
} 
Moreover, we rely on an octree representation of the voxel grid, this decreasing memory requirements significantly [21]. Thanks to the reduced computational complexity, and unlike most previous works on dense volumetric mapping that leverage on GP-GPU acceleration, we can run efficiently our reconstruction system on the $\mathrm{CPU}$, this being a beneficial trait as regards its potential implementation on the compact embedded platforms often deployed in industrial environments.

To assess quantitatively the accuracy provided by the proposed RobotFusion approach based on fusing depth measurements into a TSDF within a viewpoint and across viewpoints, we created a synthetic setup in the Gazebo simulator integrated in the ROS framework. In particular, we simulated an industrial robot equipped with an RGB-D sensor and a table-top scene. As the simulation environment provides noiseless RGB-D data, we implemented a noise model similar to that proposed by Nguyen et al. [13]. In the experiments, we compared the reconstructions provided by RobotFusion to those that one would achieve by simply stitching together the point clouds from the different vantage points into the global coordinate system of the robot, which is also straightforwardly attainable by deploying robot kinematics. Figure 2a shows that the proposed approach can achieve smooth and accurate 3D reconstructions as well as how the devised fusion process provides a significantly higher accuracy with respect to simple stitching of the point clouds. Figure 2c pertains a reconstruction experiment carried out with real data: while RobotFusion can recover the object's surface accurately, the reconstruction created by stitching together the points clouds shows many gross errors due to sensor noise. Eventually, Fig. $2 \mathrm{~b}$ concerns en experiment aimed at evaluating the benefits brought in by fusing together multiple depth images at each viewpoint: the reconstruction error tends to decrease and then stabilize as more and more depth images get fused at a single viewing position. As such, it turns out beneficial to deploy a sufficient number of images (e.g. 10-15) to effectively smooth out noise and minimize the reconstruction error.

\subsection{Plane-Based Segmentation}

Industrial robots can be mounted only in three standard positions: floor, wall, ceiling (seldom at 45 degrees). Thus, we can always calculate the relation between the gravity vector and the $z$-axis of the robot in the robot (or world) coordinate system. For example, in a floor-mounted robot the gravity vector is parallel and opposite to the $z$-axis: $\mathbf{g}=(0,0,-\mathbf{z})=(0,0,-1)$.

Hence, given a point cloud $\mathcal{P}=\left\{\mathbf{p}_{\mathbf{1}}, \ldots, \mathbf{p}_{\mathbf{n}}\right\}$ and the associated set of normals $\mathcal{N}=\left\{\mathbf{n}_{\mathbf{1}}, \ldots, \mathbf{n}_{\mathbf{n}}\right\}$, we extract the points belonging to planes orthogonal to the gravity vector by creating a $1-D$ histogram $\mathcal{H}=\left\{S_{1}, . ., S_{k}\right\}$ where each bin represents a subset (or slice) of points belonging approximately to the same plane. More precisely, assuming a floor-mounted robot and denoting the slicesize (histogram bin-size) as $\lambda$ :

$$
\mathbf{p}_{\mathbf{i}} \in S_{j} \Longleftrightarrow \mathbf{n}_{\mathbf{i}} \cdot(-\mathbf{g})<\cos (\alpha), j=\left\lfloor p_{\mathbf{z}, i} / \lambda\right\rfloor
$$




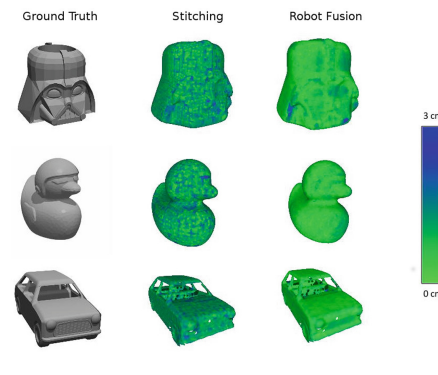

(a)

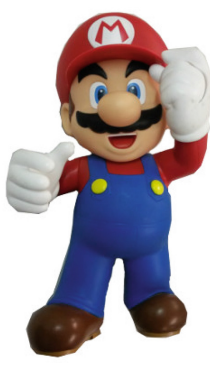

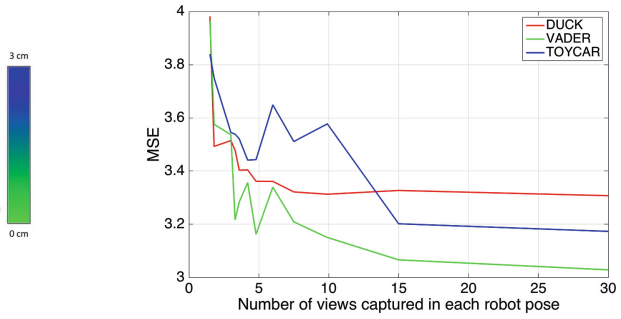

(b)
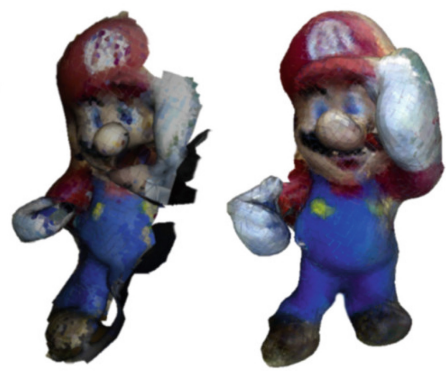

(c)

Fig. 2. (a) Synthetic data. Left: original 3D CAD model used as ground truth. Center: reconstruction by stitching point clouds. Right: TSDF-based reconstruction by RobotFusion. Colors encode the metric error w.r.t. the ground truth at reconstructed surface points. (b) Mean square error in function of the number of views for the 3 different models of Fig. 2c. The proposed TSDF-based approach obtains an increasing accuracy with a higher number of frames captured in each robot poses (in this case 12 robot poses around object). (c) Real data. Left: original 3D object. Center: reconstruction by stitching point clouds. Right: TSDF-based reconstruction by RobotFusion.

$\alpha$ being an arbitrary angular threshold used to define the maximum allowed plane inclination w.r.t the gravity vector and to withstand the presence of noise on the computed normals.

As illustrated in Fig. 3a, for a floor-mounted robot histogram $\mathcal{H}$ highlights the position along the gravity axis of possible horizontal planes. By defining $S_{\mathbf{z}_{\text {min }}, j}, S_{\mathbf{z}_{\text {max }}, j}$ as, respectively, the minimum and maximum $z$ coordinates in slice $S_{j}$, we can extract planes by selecting subsets of points belonging to those histogram bins that report a value over a pre-defined threshold. We call this approach as HeightMap segmentation.

Remarkably, plane extraction methods such as those based on $R A N S A C$ have no predictable execution time [17] due to their intrinsic randomized iterative nature. This is also the case of the 1-Point RANSAC approach [22], which enables to estimate dominant planes by means of a 1-point (rather than 3) RANSAC plane fitting by exploiting the normal associated to each point. Conversely, the proposed HeightMap approach enjoys a deterministic and fast execution time, due to its complexity being linear in the size of the point cloud. 


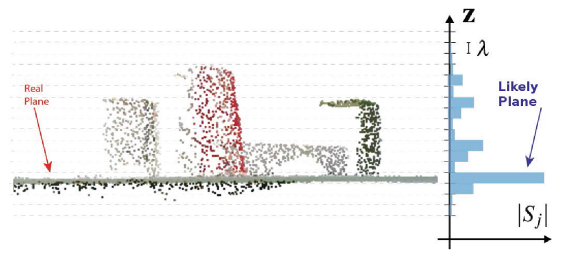

(a)

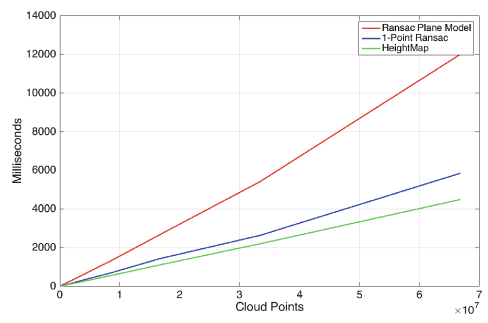

(b)

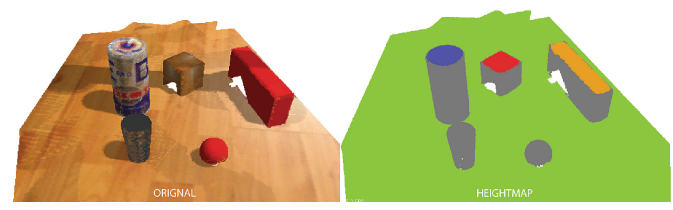

(c)

Fig. 3. (a) Side view of a point cloud representing a table-top scene, with four objects. Accordingly, the bin of the histogram reported on the right hand-side corresponding to the table is the highest one, while object surfaces tend to report much smaller bin values. (b) Execution times of plane extraction algorithms while increasing the point cloud size. (c) Example of horizontal planes extracted by a single run of the proposed HeightMap segmentation algorithm.

Figure $3 \mathrm{~b}$ reports the running times yielded by different plane extraction methods, namely HeightMap, standard RANSAC and 1-point RANSAC, in experiments carried out in the simulated working environment already described in Subsect.3.1. As we can see, HeightMap is the fastest approach and features a linear growth of the measured execution time with respect to the point cloud size. Figure $3 \mathrm{c}$ shows an example of the horizontal planes extracted by the HeightMap method from a point cloud. It is worth pointing out that a single run of the method allows to extract all the sought parallel planes, while getting an equivalent result by RANSAC-based approaches would require multiple iterations.

On a broader level, we wish to point out that HeightMap may be applied to extract planes orthogonal to any arbitrarily oriented gravity-vector. Purposely, the point cloud may require a rotation to align one axis (e.g. the $z$-axis) to the considered gravity-vector.

Under the previously highlighted assumption of objects placed on a planar support, plane extraction provides a strong cue to segment the individual objects. Indeed, the largest plane extracted by HeightMap can be quite safely assumemed to represent the workbench and thus simply removed from the point cloud. Thereby, the objects become disconnected one to another and the points belonging to each object's surface can be identified straightforwardly by standard tools such as Euclidean Clustering ${ }^{2}$.

$\overline{2}$ http://www.pointclouds.org/documentation/tutorials/cluster_extraction.php. 


\subsection{Extraction of Grasp Points}

The goal of the proposed grasp point extraction approach is to estimate directly grasp points on the object surface regardless of the gripper shape (or regardless of the number of fingers in case of a robotic hand) by means of an efficient iterative algorithm. Our main approach to reduce the complexity of grasp point estimation on a $3 \mathrm{D}$ surface is to reduce the solution space from $3 \mathrm{D}$ to $2 \mathrm{D}$, so to be able then to apply well known planar grasp algorithms [3,6,15]. Planar grasp algorithms require a $2 \mathrm{D}$ polygon as input: to deal with this, we reuse the HeightMap segmentation technique proposed in Subsect. 3.2 applied on a single object cluster rather than on the point cloud of the entire 3D scene. Figure 4a shows the proposed pipeline to compute grasp points in the robot coordinate system, which is described in the following.

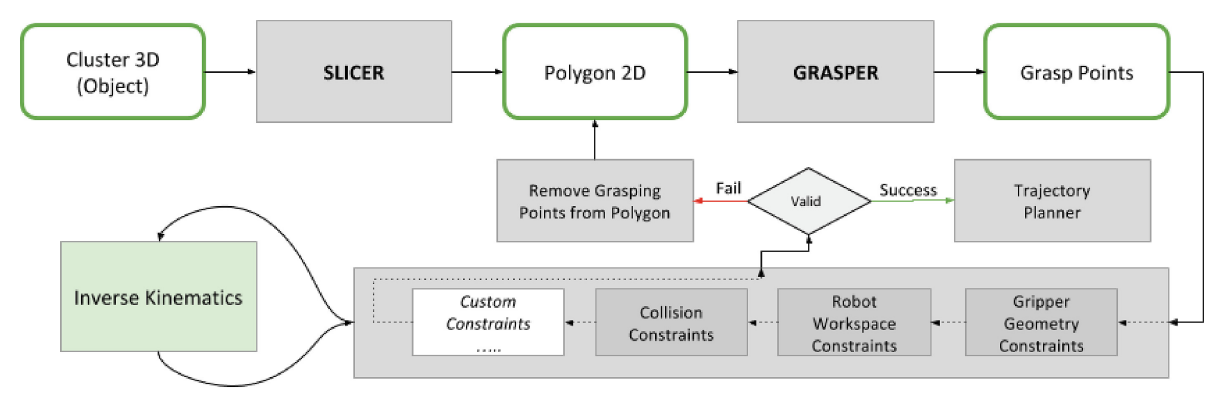

(a)
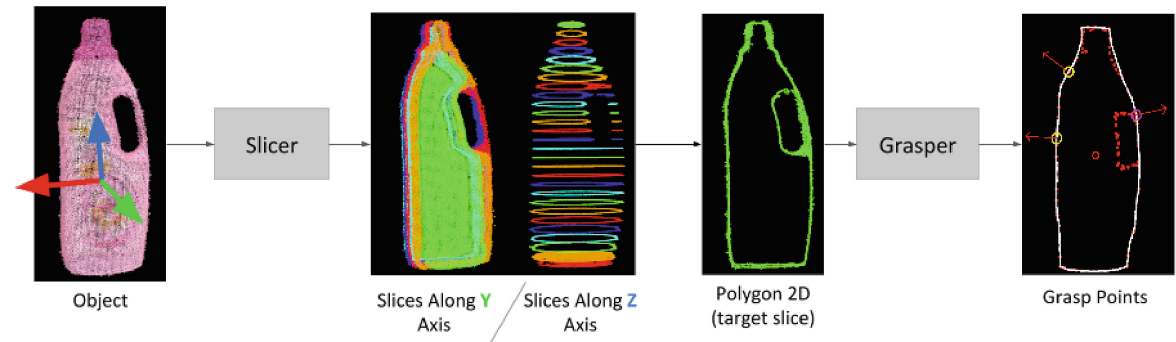

(b)

Fig. 4. (a) Proposed pipeline for grasp points extraction. Each subset of the polygon points (defined as Grasping Points) will be validated through $N$ validation stages. In addition to fixed constraints, the user can choose custom constraints for a specific task. (b) Graphical representation of a 3D object passing through the previous pipeline until it reaches a valid grasp point configuration.

Principal Axes. To begin with, each 3D object cluster is sliced along its principal axes. To obtain these stable axes, we compute the EigenValue Decomposition (EVD) of the covariance matrix of each object cluster [19], which yields three repeatable directions. 
Slicer. The algorithm selects a slice and projects the points onto a plane orthogonal to the slicing axis. To simplify the obtained shape, the system computes the concave hull of the slice, this yielding a $2 \mathrm{D}$ polygon suitable to the next stages.

Grasp Point Extraction. At this stage, $N$ points are randomly extracted from the polygon boundary (in practice, only the first points are randomly extracted, the remaining ones are chosen so to avoid useless point subsets, e.g. adjacent points).

Validation. The extracted grasp points are then evaluated through several stages. The first stage checks for geometrical constraints: if the detected grasp points lie outside of the gripper (or robotic hand) workspace, the grasp points are removed. This can be carried out, e.g., by checking if the distance between 2 grasp points is larger than the maximum opening of a parallel gripper. The second stage checks for robot workspace constraints, by evaluating whether grasp points can be reached in a dexterous portion of the workspace. The third stage evaluates collision constraints, by checking if the gripper, with the 6-DOF pose relative to the grasp points, would collide with environment. In addition, custom constraints can also be further added to refine grasp points in case of specific tasks or manipulators, by adding additional validation stages.

Grasp Point Removal. If one of the validation stages fails, the set of grasp points is removed for the current 2D polygon. Conversely, if no validation stage fails, since the grasp points are already in the robot coordinate system, the system can directly compute the full inverse kinematic chain from the grasp points to the robot base, so that a trajectory planner can be instructed to easily allow the robot approach the object and carry out the grasp.

Of course, given the greedy and iterative nature of this algorithm, the determined solution may not be the optimal one, and the system may also fail to find a feasible solution if the required iterations exceed the maximum number of allowed iterations.

\section{Grasping Experiments}

The entire pipeline comprising 3D reconstruction, plane-based segmentation and extraction of grasp points was tested in real grasping experiments dealing with 8 scenes created by placing several objects (between a set of 8) on a planar support, as illustrated in Fig. 5b. The experimental setup consists of an Industrial Robot, i.e. a Comau Smart Six with six degrees of freedom and an accuracy and repeatability lesser than $0.05 \mathrm{~mm}$. The end effector, shown in Fig. 5a, is a dualuse tool: the first part of the tool is an Asus Xtion RGB-D sensor, the second part a two finger Robotic Hand with three contact point providing a more stable grasp compared to a simple parallel gripper with only two contact points.

Each scene was tested 5 times and in each experiment the robot had to grasp all the objects present in the scene. Accordingly, a binary outcome (Success or 


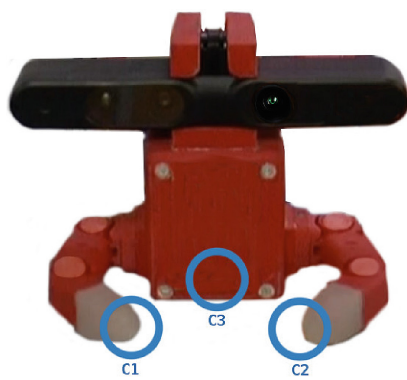

(a)

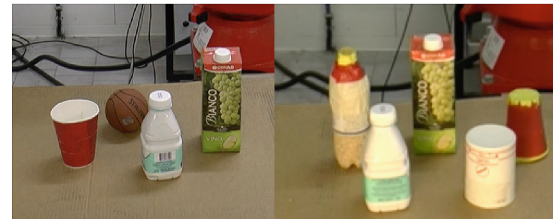

(b)

Fig. 5. (a) End Effector used during the experiments. The top part is an Asus Xtion RGB-D Sensor, the bottom part is the Gripper: a two finger robotic hand with three contacts points $(C 1, C 2, C 3)$. (b) Two sample scenes of our grasping experiments.

Table 1. Grasping results

\begin{tabular}{|c|c|c|c|}
\hline Object Type & $\begin{array}{l}\text { Grasp carried by the robot and corre- } \\
\text { sponding grasp configuration }\end{array}$ & Possible Trials & Success $(\%)$ \\
\hline Ball & & 20 & 100 \\
\hline Bottle & & 20 & 100 \\
\hline Box & & 20 & 100 \\
\hline Cup & & 30 & 95 \\
\hline Cylinder & & 20 & 100 \\
\hline Milk & & 30 & 100 \\
\hline Showergel & & 20 & 75 \\
\hline Winepack & 7 & 30 & 100 \\
\hline
\end{tabular}


Failure) was reported for each possible grasp depending on whether or not the robot succeeds in grasping the object and placing it into a bin. Thus, Success requires all the stages in our pipeline to work effectively, while a Failure may be ascribed to a variety of causes, such as missing an object because of a wrong segmentation or an unstable grasp (e.g. the robot does not picks up the object or the object falls while being moved towards the bin) due imprecise extraction of the grasping points.

Table 1 summarizes the results of our grasping experiments. In particular, for each of the 8 objects, the last two columns report the number of possible grasps within the 40 experiments ( 5 tests for each of the 8 scenes) as well as the percentage of successful ones. Each row depicts also two examples of grasps performed by the robot together with the associated contact points computed by the algorithm described in Subsect. 3.3. It is worth highlighting that, as each object was placed in the different scenes according to different poses (see the pictures in Table1), those automatically determined by our algorithm are not simple caging grasps but, indeed, stable predetermined grasp configurations. The overall success rate over all the grasping experiments turned out to be $96,56 \%$, with the failures mainly due to imprecise localization of the grasping points.

Additional results are provided in the supplementary material, which includes a video recorded during the grasping experiments.

\section{Conclusions}

We have described a practical and effective approach to 3D reconstruction and object grasping whereby, instead of registering the depth data acquired from different viewpoints with greedy algorithms, such as Iterative Closest Point (ICP), we deploy the high accuracy an repeatability of industrial robots to compute sensor poses directly by robot kinematics. To accomplish accurate $3 \mathrm{D}$ reconstruction, we rely on the TSDF representation popularized by KinectFusion and then propose original algorithms to both segment the objects out of a planar support as well as estimate automatically grasping points from the object surfaces. We have reported results aimed at highlighting the advantages brought in by each stage of the proposed pipeline as well as demonstrated the effectiveness of the overall system in real grasping experiments carried out in our Lab by an industrial robot equipped with an RGB-D camera and a two finger gripper.

\section{References}

1. Aldoma, A., Tombari, F., Prankl, J., Richtsfeld, A., Stefano, L.D., Vincze, M.: Multimodal cue integration through hypotheses verification for RGB-D object recognition and 6DOF pose estimation. In: 2013 IEEE International Conference on Robotics and Automation (ICRA), pp. 2104-2111, May 2013

2. Aldoma, A., Vincze, M., Blodow, N., Gossow, D., Gedikli, S., Rusu, R.B., Bradski, G.: CAD-model recognition and 6DOF pose estimation using 3d cues. In: 2011 IEEE International Conference on Computer Vision Workshops (ICCV Workshops), pp. 585-592, November 2011 
3. Bicchi, A., Kumar, V.: Robotic grasping and contact: a review. In: 2000 Proceedings of IEEE International Conference on Robotics and Automation, ICRA 2000, vol. 1, pp. 348-353 (2000)

4. Bylow, E., Sturm, J., Kerl, C., Kahl, F., Cremers, D.: Real-time camera tracking and $3 \mathrm{~d}$ reconstruction using signed distance functions. In: Robotics: Science and Systems (RSS), Online Proceedings (2013)

5. Engel, J., Schöps, T., Cremers, D.: LSD-SLAM: large-scale direct monocular SLAM. In: Fleet, D., Pajdla, T., Schiele, B., Tuytelaars, T. (eds.) ECCV 2014. LNCS, vol. 8693, pp. 834-849. Springer, Heidelberg (2014). doi:10.1007/ 978-3-319-10605-2_54

6. Ferrari, C., Canny, J.: Planning optimal grasps. In: Proceedings of 1992 IEEE International Conference on Robotics and Automation, vol. 3, pp. 2290-2295, May 1992

7. Fulhammer, T., Aldoma, A., Zillich, M., Vincze, M.: Temporal integration of feature correspondences for enhanced recognition in cluttered and dynamic environments. In: International Conferenec on Robotics and Automation (ICRA), pp. 3003-3009, May 2015

8. Hang, K., Stork, J., Kragic, D.: Hierarchical fingertip space for multi-fingered precision grasping. In: 2014 IEEE/RSJ International Conference on Intelligent Robots and Systems (IROS 2014), pp. 1641-1648, September 2014

9. ISO TC 184SC 2 Robots, robotic devices: ISO 9283. Manipulating industrial robots- Performance criteria and related test methods. International Organization for Standardization, Geneva, Switzerland (2015). http://www.iso.org

10. Kehl, W., Navab, N., Ilic, S.: Coloured signed distance fields for full 3d object reconstruction. In: Proceedings of the British Machine Vision Conference. BMVA Press (2014)

11. Montana, D.: The condition for contact grasp stability. In: IEEE International Conference on Robotics and Automation (1991)

12. Newcombe, R.A., Davison, A.J., Izadi, S., Kohli, P., Hilliges, O., Shotton, J., Molyneaux, D., Hodges, S., Kim, D., Fitzgibbon, A.: KinectFusion: real-time dense surface mapping and tracking. In: 10th IEEE International Symposium on Mixed and Augmented Reality, pp. 127-136, October 2011. http://ieeexplore.ieee.org/ lpdocs/epic03/wrapper.htm?arnumber $=6162880$

13. Nguyen, C., Izadi, S., Lovell, D.: Modeling kinect sensor noise for improved 3d reconstruction and tracking. In: 2012 Second International Conference on 3D Imaging, Modeling, Processing, Visualization and Transmission (3DIMPVT), pp. 524530, October 2012

14. Papazov, H., Parusel, K., Krieger, B.: Rigid 3d geometry matching for grasping of known objects in cluttered scenes. Int. J. Robot. Res. 31, 538-553 (2012)

15. Ponce, J., Faverjon, B.: On computing three-finger force-closure grasps of polygonal objects. In: Fifth International Conference on Advanced Robotics, Robots in Unstructured Environments, ICAR 1991, vol. 2, pp. 1018-1023, June 1991

16. Popovi, M., Kraft, D., Bodenhagen, L., Baeski, E., Pugeault, N., Kragic, D., Asfour, T., Krger, N.: A strategy for grasping unknown objects based on coplanarity and colour information. Robot. Auton. Syst. 58(5), 551-565 (2010)

17. Raguram, R., Frahm, J.-M., Pollefeys, M.: A comparative analysis of RANSAC techniques leading to adaptive real-time random sample consensus. In: Forsyth, D., Torr, P., Zisserman, A. (eds.) ECCV 2008. LNCS, vol. 5303, pp. 500-513. Springer, Heidelberg (2008). doi:10.1007/978-3-540-88688-4_37 
18. Garrido-Jurado, S., Muoz-Salinas, R., Madrid-Cuevas, F.J., Marn-Jimnez, M.J.: Automatic generation and detection of highly reliable fiducial markers under occlusion. Pattern Recogn. 47(6), 2280-2292 (2014)

19. Salti, S., Tombari, F., Stefano, L.D.: Shot: unique signatures of histograms for surface and texture description. Comput., Vis. Image Underst. 125, 251-264 (2014). http://www.sciencedirect.com/science/article/pii/S1077314214000988

20. Saut, J.P., Sidobre, D.: Efficient models for grasp planning with a multi-fingered hand. Robot. Autonom. Syst. 60(3), 347-357 (2012). http://www.sciencedirect.com/science/article/pii/S0921889011001515, Autonomous Grasping

21. Steinbrucker, F., Kerl, C., Cremers, D.: Large-scale multi-resolution surface reconstruction from RGB-D sequences. In: The IEEE International Conference on Computer Vision (ICCV), December 2013

22. Tombari, F., Fioraio, N., Cavallari, T., Salti, S., Petrelli, A., Stefano, L.D.: Automatic detection of pole-like structures in 3d urban environments. In: 2014 IEEE/RSJ International Conference on Intelligent Robots and Systems (IROS 2014), pp. 4922-4929, September 2014

23. Whelan, T., Kaess, M., Johannsson, H., Fallon, M., Leonard, J.J., Mcdonald, J.: Real-time large scale dense RGB-D SLAM with volumetric fusion. Int. J. Robot. Res. IJRR 34, 598-626 (2014)

24. Xie, Z., Singh, A., Uang, J., Narayan, K.S., Abbeel, P.: Multimodal blending for high-accuracy instance recognition. In: 2013 IEEE/RSJ International Conference on Intelligent Robots and Systems (IROS), pp. 2214-2221, November 2013

25. Jiang, Y., Moseson, S., Saxena, A.: Efficient grasping from RGBD images: learning using a new rectangle representation. In: 2011 IEEE International Conference on Robotics and Automation (ICRA) (2011) 